\title{
Neurotrophins Elevate cAMP to Reach a Threshold Required to Overcome Inhibition by MAG through Extracellular Signal-Regulated Kinase-Dependent Inhibition of Phosphodiesterase
}

\author{
Ying Gao, ${ }^{\star}$ Elena Nikulina, ${ }^{\star}$ Wilfredo Mellado, and Marie T. Filbin \\ Department of Biological Sciences, Hunter College, City University of New York, New York, New York 10021
}

\begin{abstract}
Inhibitors of regeneration in myelin, such as myelin-associated glycoprotein (MAG), play an important role in preventing regeneration after CNS injury. Elevation of cAMP, either with dibutyryl-cAMP (db-cAMP) or by priming with a variety of neurotrophins, overcomes inhibition by MAG and myelin. However, activation of cAMP is not generally regarded as a signaling pathway for neurotrophins. Here we show that the NGF-like neurotrophins overcome inhibition by MAG by activating tyrosine kinase receptors. We also show that activation of extracellular signal-regulated kinase (Erk) by BDNF is required to overcome inhibition by MAG, and that activated Erk transiently inhibits phosphodiesterase 4 (PDE4), the enzyme that hydrolyzes cAMP. Inhibition of PDE4 then allows cAMP to increase and so initiates the pathway to overcome inhibition. Furthermore, we also show that basal levels of Erk activation and basal cAMP levels contribute to the effects of db-cAMP by pushing the combined levels of cAMP above a threshold required to overcome inhibition. Together, these results not only show how NGF-like neurotrophins can elevate cAMP and overcome inhibition but also point to a novel mechanism of cross talk in neurons from the Erk to the cAMP signaling pathways.
\end{abstract}

Key words: regeneration; myelin-associated glycoprotein; neurotrophin; Erk; phosphodiesterase; cAMP

\section{Introduction}

The lack of regeneration after injury to the adult mammalian CNS is largely attributable to a glial scar (Fitch and Silver, 1999) and inhibitors in myelin (Qiu et al., 2000; Filbin, 2003). To date, three myelin inhibitors of regeneration have been identified. These are myelin-associated glycoprotein (MAG) (McKerracher et al., 1994; Mukhopadhyay et al., 1994), NogoA (Chen et al., 2000; GrandPre et al., 2000), and recently, oligodendrocyte myelin glycoprotein (OMgp) (Wang et al., 2002b). Surprisingly, MAG and OMgp and one Nogo domain, Nogo66, interact with the same receptor, the Nogo receptor (Fournier et al., 2001; Domeniconi et al., 2002; Liu et al., 2002; Wang et al., 2002b). Moreover, the signaling partner for this receptor is the p75 neurotrophin receptor (p75NTR) (Wang et al., 2002a). There are two approaches, then, to overcome inhibitors in myelin and encourage regeneration. Either the environment can be changed by blocking the inhibitors or their common receptor, or the intrinsic growth capacity of the neuron-axon can be altered such that it

\footnotetext{
Received July 21, 2003; revised Aug. 28, 2003; accepted Sept. 2, 2003.

This work was supported by grants from the New York State Spinal Cord fund, National Institutes of Health (NIH) Grant NS 37060, grants from the National Multiple Sclerosis Society, and core facility grants from the Research Centers for Minorities Institute-NIH and Specialized Neuroscience Research Programs-NIH (NS41073). We thank Marie Handler and George Harter for their outstanding technical support and Dr. Lloyd Williams for his help with the image analysis.

*Y.G. and E.N. contributed equally to this study.

Correspondence should be addressed to Dr. Marie T. Filbin, Biology Department, Hunter College, 695 Park Avenue, New York, NY 10021. E-mail: Filbin@genectr.hunter.cuny.edu.

Copyright $\odot 2003$ Society for Neuroscience $\quad$ 0270-6474/03/2311770-08\$15.00/0
}

ignores the inhibitory molecules. Taking the latter approach, success with two treatments that change the growth capacity of the axon-neuron and allow growth on inhibitors of myelin has been reported. First, if the small G-protein Rho is inactivated in axons, regeneration occurs both in vitro and in vivo (Lehmann et al., 1999). Second, if neuronal cAMP is elevated, axons are no longer inhibited not only by MAG but also by myelin in general (Song et al., 1998; Cai et al., 1999). Importantly, elevation of cAMP in the cell body results in regeneration of lesioned spinal axons (Neumann et al., 2002; Qiu et al., 2002).

cAMP was elevated in a variety of neurons either with an analog, dibutyryl-cAMP (db-cAMP), or by exposure of the neurons to neurotrophins before exposure to the inhibitor(s). For cerebellar neurons, "priming" with BDNF or glial cell linederived neurotrophic factor (GDNF), but not NGF, overcame inhibition by MAG and myelin, whereas for dorsal root ganglion (DRG) neurons, any one of these three neurotrophins was effective (Cai et al., 1999). The cAMP pathway is not generally regarded as a pathway activated by neurotrophins. Rather, the Rasextracellular signal-regulated kinase (Erk) pathway is activated by neurotrophins that play a role in survival and differentiation (Kaplan and Miller, 2000). However, in non-neuronal cells, activated Erk has been shown to phosphorylate and inhibit a subfamily of the group of enzymes responsible for cAMP degradation, phosphodiesterases 4 (PDE4s) (Hoffmann et al., 1999; Baillie et al., 2000; MacKenzie et al., 2000). The PDE4 subfamily represents the most abundant PDEs in neuronal tissue $(\sim 70 \%)$ (Jin et al., 
1999). They are cAMP specific and are specifically inhibited by the drug rolipram (Houslay and Kolch, 2000). Here we show that neurotrophins elevate cAMP by an Erk-dependent inhibition of PDE, and that a threshold of CAMP-PKA activation is required for both BDNF and db-cAMP to overcome inhibition by MAG. These results identify the early steps in the neurotrophinsignaling pathway that overcomes inhibition by MAG and also point to a novel mechanism of cross talk between the Erk and the cAMP signaling pathways in neurons.

\section{Materials and Methods}

Priming neurons with neurotrophins. Isolated cerebellar [postnatal day 3 (P3)-P7] or DRG (P5-P8) neurons in Sato (20 nM progesterone, $30 \mathrm{~nm}$ selenium, $5 \mu \mathrm{g} / \mathrm{ml}$ insulin, $4 \mathrm{mg} / \mathrm{ml} \mathrm{BSA}, 0.1 \mu \mathrm{g} / \mathrm{ml} \mathrm{L}$-thyroxine, and 0.08 $\mu \mathrm{g} / \mathrm{ml}$ tri-iodo-thyronine) were plated onto the poly-L-lysine-coated 24 well plates at a density of $1 \times 10^{6}$ cells/well (Cai et al., 1999). Where indicated, BDNF, GDNF, NGF (200 ng/ml), rolipram $(0.1$ or $0.5 \mu \mathrm{M})$ (Sigma, St. Louis, MO), or forskolin $(0.1-10 \mu \mathrm{M}$ ) (Calbiochem, La Jolla, CA) were added in the presence or absence of the tyrosine kinase (Trk) inhibitor K252a (50 nм) (Calbiochem), functional blocking antibody of p75 (kindly provided by Dr. Moses V. Chao, Skirball Institute, New York University, New York, NY), the PKA inhibitor KT-5720 (200 nM) (Calbiochem), or the mitogen-activated protein kinase kinase (MEK) inhibitors 1,4diamino-2,3-dicyano-1,4-bis(aminophenylthio)buta-diene(U0126) (5 $\mu \mathrm{M})$ and 2-(2'-amino-3'-methoxyphenyl)-oxanaphtha-len-4-one (PD98059) $(50 \mu \mathrm{M})($ Calbiochem). After overnight culture, the media was removed and neurons were washed with PBS and removed with $0.1 \%$ trypsin. Trypsinization was stopped by adding $5 \mathrm{ml}$ of DMEM containing $10 \%$ FCS. Neurons were centrifuged at $800 \mathrm{rpm}$ for $6 \mathrm{~min}$, resuspended in Sato, and plated immediately onto either MAG-expressing Chinese hamster ovary (CHO) cells or control CHO cells.

Neurite outgrowth assay. Monolayers of control and MAG-expressing $\mathrm{CHO}$ cells were grown to confluency in individual chambers of an eight well tissue culture slide (Lab-Tek). The neurite outgrowth assay was performed as described previously (Mukhopadhyay et al., 1994; Cai et al., 1999) by adding $2 \times 10^{4}$ cerebellar or DRG neurons, either primed or unprimed, to the $\mathrm{CHO}$ cell monolayers. Where indicated, db-cAMP (1 $\mathrm{mM}$ ) with or without the PKA inhibitor KT-5720 (200 nM) or the MEK inhibitor PD98059 $(50 \mu \mathrm{M})$ was added to the cultures. After 16-18 hr, the cultures were fixed and immunostained for growth-associated protein-43 (GAP43) as described previously (Mukhopadhyay et al., 1994). The length of the longest neurite or the total process length for each GAP43-positive neuron for the first 200 neurons encountered when scanning the slide in a systematic manner was determined using an Oncor (Gaithersburg, MD) image analysis program. Using a video camera, the neuron image was projected to a computer screen. The longest neurite or entire processes were traced and quantitated.

Western blot analysis. Neurons were starved for 2-6 hr in DMEM and then incubated for different times with combinations of BDNF (200 $\mathrm{ng} / \mathrm{ml})$, NGF (200 ng/ml), db-cAMP (1 mM), the Trk receptor inhibitor K252a (50 nM), the MEK inhibitors PD98059 (50 $\mu \mathrm{M})$ and U0126 (5 $\mu \mathrm{M})$, or the PKA inhibitors H89 (5 $\mu \mathrm{M})$ and KT-2750 (200 nM) (Calbiochem) as indicated. Neurons were then washed with PBS and lysed in sample buffer ( $62.5 \mathrm{~mm}$ Tris- $\mathrm{HCl}$, pH 6.8, and 2\% SDS) that included phosphatase inhibitors, $20 \mathrm{~mm} \mathrm{NaF}, 1 \mathrm{~mm} \quad \mathrm{Na}_{3} \mathrm{VO}_{4}$, and $10 \mathrm{~mm}$ $\beta$-glycerophosphate (Sigma). Samples were run through DNA shredders (Qiagen, Valencia, CA), and protein was measured by a Lowry-based protein assay (Bio-Rad, Richmond, CA). For detection of Erk, $20 \mu \mathrm{g}$ of protein from the samples was loaded and separated by $10 \%$ SDS-PAGE. For detection of Trk, $50 \mu \mathrm{g}$ of samples was separated by $5 \%$ SDS-PAGE. After electrophoresis, gels were transferred to polyvinylidene difluoride membranes (NEN, Boston, MA) and probed with either a phospho-Trk (Tyr 674/675) antibody (1:750) or a phospho-Erk (Thr 202/Tyr 204) antibody $(1: 2000)$ overnight at $4^{\circ} \mathrm{C}$. Blots were incubated with an antirabbit IgG-HRP secondary antibody (1:5000) for $1 \mathrm{hr}$, and detection was performed using ECL (Amersham Biosciences, Arlington Heights, IL). The same blot was later stripped with stripping buffer $(0.2 \mathrm{M}$ glycine, $\mathrm{pH}$
2.2, $1 \%$ Tween 20 , and $0.1 \%$ SDS) for $1 \mathrm{hr}$ at room temperature and reprobed with either a Trk antibody (1:1000) or an Erk antibody (1:2000) as indicated. All antibodies were purchased from Cell Signaling.

$P D E$ assay. For each assay, $5 \times 10^{6}$ cerebellar neurons were plated per well of a poly-L-lysine coated six well dish. The neurons were incubated overnight in Sato and then starved for $2 \mathrm{hr}$ in DMEM before the addition of BDNF with or without the MEK inhibitor U0126 (5 $\mu \mathrm{M})$ for 5, 10, 30, or $60 \mathrm{~min}$. The neurons were washed once with PBS and immediately lysed on ice and scraped using a rubber policeman in $100 \mu \mathrm{l}$ of PDE buffer, 50 mm Tris-HCl, pH 7.5, 8.3 mM $\mathrm{MgCl}_{2}, 1.7$ mM EGTA, $3.75 \mathrm{~mm}$ of $\beta$-mercaptoethanol, proteinase inhibitors (Calbiochem), and phosphatase inhibitors. PDE activity was measured in lysates using a scintillation proximity assay, according to the manufacturer's instructions (Amersham Biosciences). To determine the percentage of rolipramsensitive PDE activity in the lysates, $25 \mu$ l of lysate treated with BDNF was incubated with $100 \mu \mathrm{M}$ rolipram for $15 \mathrm{~min}$ before measuring PDE activity.

cAMP assay. For each assay, $2 \times 10^{6}$ cerebellar neurons (P5-P7) were plated per well of a poly-L-lysine-coated 24 well plate. Each sample was performed in duplicate. The neurons were incubated overnight in Sato, and BDNF $(200 \mathrm{ng} / \mathrm{ml})$ was added with or without the MEK inhibitor U0126 $(5 \mu \mathrm{M})$ and incubated for an additional $30 \mathrm{~min}$. Neurons were washed with PBS and lysed in $250 \mu \mathrm{l}$ of $0.1 \mathrm{~N} \mathrm{HCl}$ by incubating first at $-20^{\circ} \mathrm{C}$ for $15 \mathrm{~min}$, then at room temperature for $15 \mathrm{~min}$, and also by vigorous pipetting. Cell lysates were collected and centrifuged at 13,000 rpm for $15 \mathrm{~min}$ at room temperature. Ten microliters of supernatant was used in a protein assay (Bio-Rad). The remaining supernatant was then acetylated, and cAMP was measured using an enzyme immunoassay, according to the manufacturer's instructions (Biomol, Plymouth Meeting, PA).

Recombinant adenovirus containing dominant negative Erk. Recombinant adenovirus was constructed by subcloning Erk 2 dominant negative cDNA (provided by Dr. Melanie Cobb, University of Texas Southwestern Medical Center at Dallas, Dallas, TX) (Robinson et al., 1996) into pTRACK CMV and finally inserted by homologous recombination into pAdeasy-1 (He et al., 1998). The viral preparation and purification was performed as described previously (He et al., 1998). DRG cells were dissociated and plated onto a tissue culture dish and incubated for $2 \mathrm{hr}$. Unadhered cells, mostly neurons, were then gently washed off and plated onto a poly-L-lysine-coated dish and incubated for an additional $2 \mathrm{hr}$. Virus in Sato medium at $100 \mathrm{pfu} /$ neuron was then added and incubated with the neurons for $60 \mathrm{~min}$, after which they were washed and the media replaced. After an additional $16 \mathrm{hr}$, neurons were then transferred to monolayers of $\mathrm{CHO}$ cells and incubated as described above. Neurite length was measured only from those neurons that were positive for both GAP43 and green fluorescent protein (GFP); in this way only infected neurons were assessed.

PKA assay. For each assay, $10^{7}$ cerebellar neurons were plated per well of a poly-L-lysine-coated six well dish. The neurons were incubated overnight in Sato and then pretreated with or without the MEK inhibitor $\mathrm{U} 0126(5 \mu \mathrm{M})$ for $1 \mathrm{hr}$ before addition of db-cAMP $(0.5,1$, or $2 \mathrm{mM})$ for $20 \mathrm{~min}$. The neurons were washed three times with PBS and immediately lysed on ice and scraped using a rubber policeman in $100 \mu \mathrm{l}$ of lysis buffer, 125 mM Tris-HCl, pH 8, 5\% (octylphenoxy)polyethoxyethanol (Sigma), and proteinase inhibitors (Calbiochem). Cell lysates were collected and centrifuged at $13,000 \mathrm{rpm}$ for $10 \mathrm{~min}$ at $4^{\circ} \mathrm{C}$. Ten microliters of supernatant was used in a protein assay (Bio-Rad). PKA activity was measured in $10 \mu \mathrm{g}$ of lysates using a PepTag assay for nonradioactive detection of cAMP-dependent protein kinase activity, according to the manufacturer's instructions (Promega, Madison, WI).

\section{Results \\ BDNF and NGF signal through Trk receptors and not the p75 receptor to overcome inhibition by MAG}

We have shown previously that priming cerebellar neurons with BDNF and GDNF but not NGF overcame inhibition, whereas priming DRG neurons with any one of these three neurotrophins 
accomplished the same effect (Cai et al., 1999). Now we wanted to determine whether, for BDNF and NGF, this effect was through the Trk-like NGF receptors or through the p75NTR. NGF has no effect on the inhibition of cerebellar neurons by MAG (Cai et al., 1999), which express p75NTR but not TrkA (Martin-Zanca et al., 1990; Holtzman et al., 1992), suggesting that the priming effect is via Trk receptors. To address this directly, we used the Trk-specific kinase inhibitor K252a. Before testing $\mathrm{K} 252 \mathrm{a}$ in the neurite outgrowth assay, we ensured that under the conditions used here Trk receptors were indeed activated by BDNF or NGF, and that K252a blocked their activation (results not shown).

When K252a was included during priming of cerebellar neurons with BDNF or priming of DRG neurons with NGF, the block of inhibition by MAG of these neurotrophins was abrogated (Fig. 1a,b). The effect is specific for Trk receptors, because K252a has no effect on the ability of priming with GDNF to overcome inhibition by MAG (Fig. 1a). In addition, K252a has no effect on the ability of db-cAMP to overcome inhibition of cerebellar neurons (Fig. 1a). This also demonstrates that K252a is not having a more general effect on kinase inhibition. Also, as shown before, priming cerebellar neurons with NGF, with or without K252a, has no effect on inhibition by MAG.

Finally, the conclusion that Trks rather than p75NTR are involved in the neurotrophin priming effect is supported by the fact that a p75NTR antibody, at a concentration known to block neurotrophin binding (Huber and Chao, 1995), has no effect on priming with NGF or BDNF (Fig. 1c). Together, these results demonstrate that BDNF and NGF are overcoming inhibition by MAG by activating the TrkB and TrkA receptors, respectively, and not through p75NTR.

\section{Inhibitors of MEK block both the BDNF and db-cAMP effect on inhibition by MAG}

A well established pathway that is downstream from Trk activation is the Ras-Erk pathway, which plays a role in the survival effects of neurotrophins (Grewal et al., 1999; Kaplan and Miller, 2000). To determine whether Erk is activated under the conditions used here by BDNF and db-cAMP, cerebellar neurons were treated with either BDNF or different concentrations of $\mathrm{db}$ cAMP for various lengths of time before being lysed and subjected to PAGE and Western blotting, and before membranes were stained with an antibody against activated (phosphorylated) Erk. Figure $2 a$ shows that as has been reported previously, incubation of neurons with BDNF results in activation of Erk. Within 10 min of BDNF treatment, Erk is phosphorylated, and this activation is sustained for at least $120 \mathrm{~min}$. The effect is specific, because an inhibitor of MEK that is responsible for Erk activation blocks this phosphorylation. Staining the same membrane, after stripping, with an antibody against total Erk shows that equal amounts of protein were loaded on the gel, and that only the phosphorylation state changes.

In the neuronal cell line PC12, it has been shown that dbcAMP can activate Erk, albeit at a low level (Vossler et al., 1997; Bouschet et al., 2003). At a concentration of $1 \mathrm{~mm} \mathrm{db}$-cAMP, the concentration we routinely use to overcome inhibition, no de- tectable increase in activation of Erk was observed (Fig. 2b). However, when the db-cAMP is increased to 2 or $5 \mathrm{~mm}$, approximately a twofold increase in Erk activation was apparent (Fig. $2 b)$.

To assess whether activation of Erk is required for BDNF and db-cAMP to overcome inhibition by MAG, priming with BDNF was performed in the presence of either U0126 or PD98059, two MEK inhibitors, or the inhibitors were added directly to the media with db-cAMP. As shown in Figure $2 c$, each of these inhibitors abrogated the ability of both BDNF and $1 \mathrm{~mm}$ db-cAMP to overcome inhibition by MAG. The ability of $1 \mathrm{~mm}$ db-cAMP to overcome inhibition by MAG without inducing any detectable activation of Erk is likely to be a sensitivity problem. That is to say, in studies with PC12 cells in which activation of Erk by $1 \mathrm{~mm} \mathrm{db}$ cAMP was demonstrated, the cells were serum starved for $24 \mathrm{hr}$ before the assay. We found that the maximum length of time that primary neurons were able to tolerate serum deprivation and remain healthy was $\sim 6 \mathrm{hr}$. As a consequence, for primary neurons, the basal levels of Erk activation could not be reduced to zero, as was the case for PC12 cells. Therefore, on this high background, any small increases in Erk activation in response to $1 \mathrm{~mm}$ db-cAMP would be undetectable. However, the main signaling pathway of cAMP is via activation of PKA, but at $1 \mathrm{mM} \mathrm{db}$-cAMP, both a PKA and an Erk inhibitor can each block the ability to overcome inhibition. Therefore, we asked whether increasing the db-cAMP concentration would increase the signal through PKA such that the Erk signaling would not be required and inhibition would be overcome, even in the presence of an Erk inhibitor. To assess whether this was the case, the db-cAMP concentration in the assay was varied from 0.1 to $4 \mathrm{mM}$. As we reported previously, db-cAMP overcomes inhibition by MAG in a dose-dependent manner, and at a concentration of $1 \mathrm{~mm}$, inhibition by MAG is completely blocked. However, when the MEK inhibitor PD98059 is included in the assay, the dose-response curve for db-cAMP is shifted to the right (Fig. $3 a$ ). Consistent with what is shown in Figure $2 c$, in the presence of U0126, db-cAMP at $1 \mathrm{mM}$ does not overcome inhibition by MAG. However, at a concentration of 1.5-2 mM db-cAMP, inhibition by MAG is completely blocked, even in the presence of the MEK inhibitor. These results suggest that increasing the activation of PKA by increasing the db-cAMP concentration compensates for the inhibition of the Erk pathway and is sufficient, alone, to overcome inhibition. Consistent with 
a

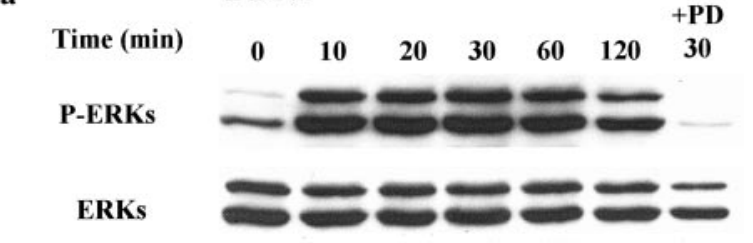

b

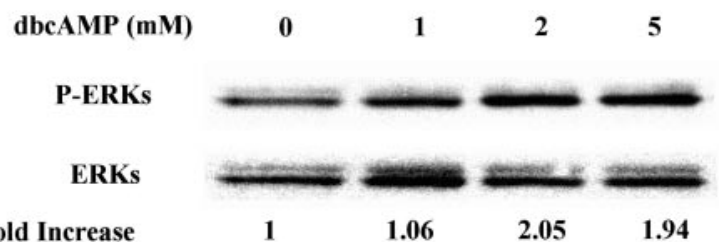

Fold Increase

1

c

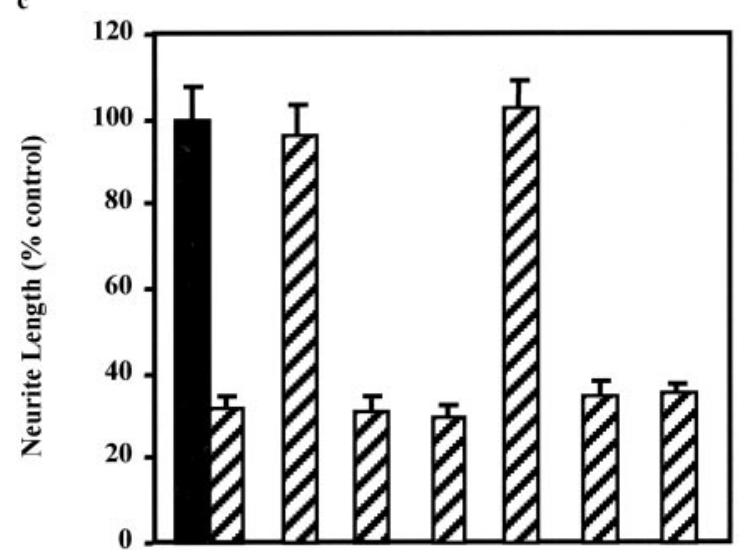

$\begin{array}{cccccccc}\text { BDNF } & - & + & + & + & - & - & - \\ \underset{\text { dbCAMP }}{\text { U0126 }} & - & - & - & - & + & + & + \\ \text { PD98059 } & - & - & + & - & - & + & - \\ & - & - & - & + & - & - & +\end{array}$

Figure 2. Inhibition of Erk blocks the ability of BDNF and db-cAMP to overcome inhibition by MAG. Cerebellar neurons were incubated with BDNF ( $200 \mathrm{ng} / \mathrm{ml})$ for various times $(a)$ or with $\mathrm{db}$-cAMP at different concentrations (1-5 mM) (b) before being lysed, subjected to Western blotting, and probed for activated Erk (P-ERKS). The blots were stripped and reprobed for total Erk (ERKs). Where indicated, the MEK inhibitor PD98059 (PD; $50 \mu \mathrm{M}$ ) was included during incubation. c, Cerebellar neurons were primed with and without BDNF (200 ng/ml) before transfer to control $\mathrm{CHO}$ cells (black bars) or MAG-expressing $\mathrm{CHO}$ cells (stripped bars), or dbCAMP was added directly to the cultures at $1 \mathrm{~mm}$. Where indicated, the MEK inhibitors U0126 (5 $\mu \mathrm{m})$ or PD98059 $(50 \mu \mathrm{m})$ were included during priming or added directly to the cultures with $\mathrm{db}$-cAMP. After overnight culture, the cells were fixed and immunostained for GAP43. Results are the mean \pm SEM length of the longest neurite per neuron for $180-200$ individual neurons, standardized to percentage of control. Each experiment was performed in duplicate and repeated at least three times.

this, the activity of PKA increases as the db-cAMP concentration is increased and is inhibited slightly but significantly by U0126, indicating that PKA activation at lower $\mathrm{db}$-cAMP concentrations is a consequence of both the endogenous CAMP and the exogenous db-cAMP (Fig. 3b)

Dominant negative Erk blocks the ability of BDNF and of 1 mM db-cAMP to overcome inhibition by MAG

To complement the use of these pharmacological inhibitors of Erk activity we used a dominant negative form of Erk, which is mutated at lysine 52 in subdomain II, creating a nonproductive binding mode for ATP (Robinson et al., 1996). The dominant negative Erk was introduced into the neurons via an adenoviral a

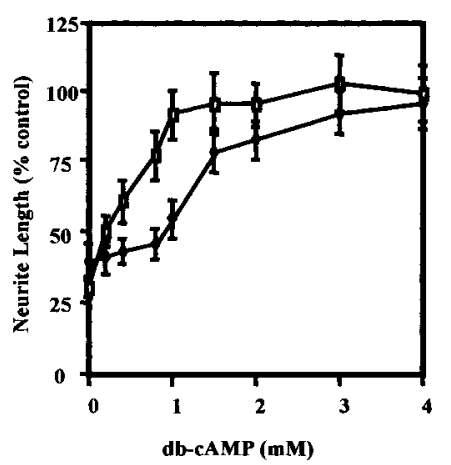

b

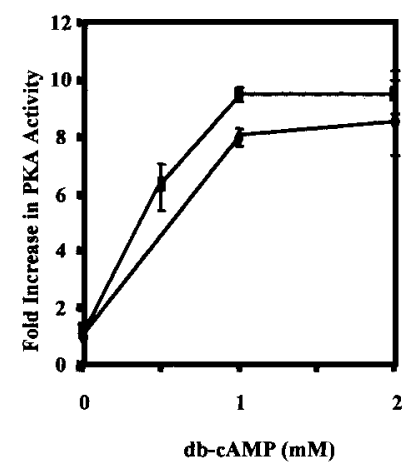

Figure 3. A threshold of CAMP-PKA activity must be reached to overcome inhibition by MAG. $a$, Db-cAMP (0-4 mM) was added directly to cultures of cerebellar neurons on MAGexpressing $\mathrm{CHO}$ cells or control $\mathrm{CHO}$ cells, with (black diamonds) and without (open squares) the MEK inhibitor U0126 (5 $\mu \mathrm{m})$. After overnight culture, the cells were fixed and immunostained for GAP43. Results are the mean \pm SEM length of the longest neurite per neuron for 180-200 individual neurons, standardized to percentage of length on control $\mathrm{CHO}$ cells. Each experiment was performed in duplicate and repeated at least three times. $b, \mathrm{db}$-CAMP $(0-2 \mathrm{~mm})$ was added to cerebellar neurons plated on poly-L-lysine, preincubated with (black diamonds) and without (open squares) U0126 (5 $\mu \mathrm{m}$ ) for $60 \mathrm{~min}$. After an additional $20 \mathrm{~min}$, cells were lysed and PKA activity was measured. Results are from four experiments, each in quadruplicate.

$\mathbf{a}$ b
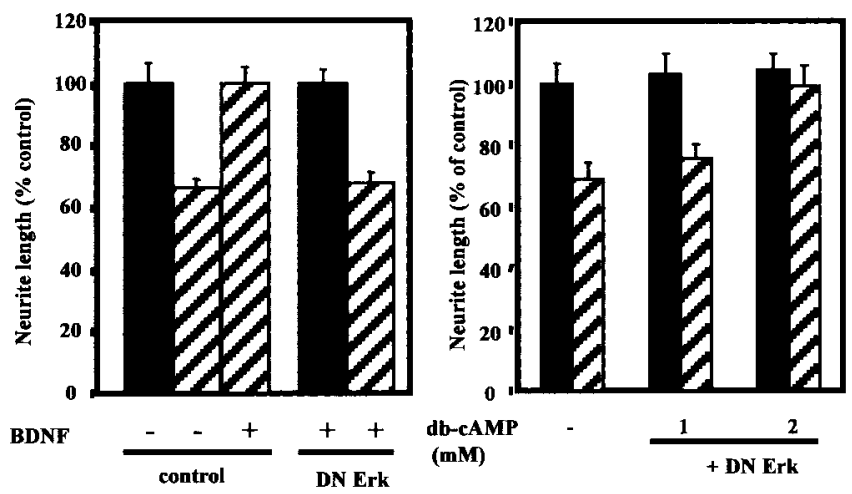

Figure 4. Dominant negative Erk (DN Erk) blocks the ability of BDNF and low but not high concentrations of db-cAMP to overcome inhibition by MAG. DRG neurons on poly-L-lysine were infected with an adenoviral vector containing dominant negative Erk and a GFP, or with a vector with only GFP as a control. Infected neurons were either primed overnight with BDNF before being transferred to the MAG (black bars) and control (stripped bars) monolayers ( $a$ ) or transferred directly to the monolayers, with and without 1 or $2 \mathrm{~mm}$ db-CAMP $(b)$. After overnight culture, the cells were fixed and immunostained for GAP43. Results are the mean \pm SEM length of the longest neurite per neuron for 180-200 individual neurons positive for both GAP43 and GFP, standardized to percentage of control. Each experiment was performed in duplicate and repeated at least three times.

delivery system. The adenovirus also carries GFP, allowing infected neurons to be distinguished from uninfected neurons. As shown in Figure 4, for DRG neurons infected with the dominant negative Erk virus, neither BDNF nor $1 \mathrm{~mm}$ db-cAMP blocked inhibition. In contrast, even in the presence of dominant negative Erk, $2 \mathrm{~mm} \mathrm{db}$-cAMP overcomes inhibition by MAG. Dominant negative Erk had no effect on inhibition by MAG in the absence of BDNF or db-cAMP. These results are consistent with the results above using the pharmacological agents in that they also indicate that the ability of BDNF to block inhibition is entirely dependent on the activation of Erk, whereas this is true for the db-cAMP effect only at $1 \mathrm{~mm}$ and not at $2 \mathrm{~mm}$. 


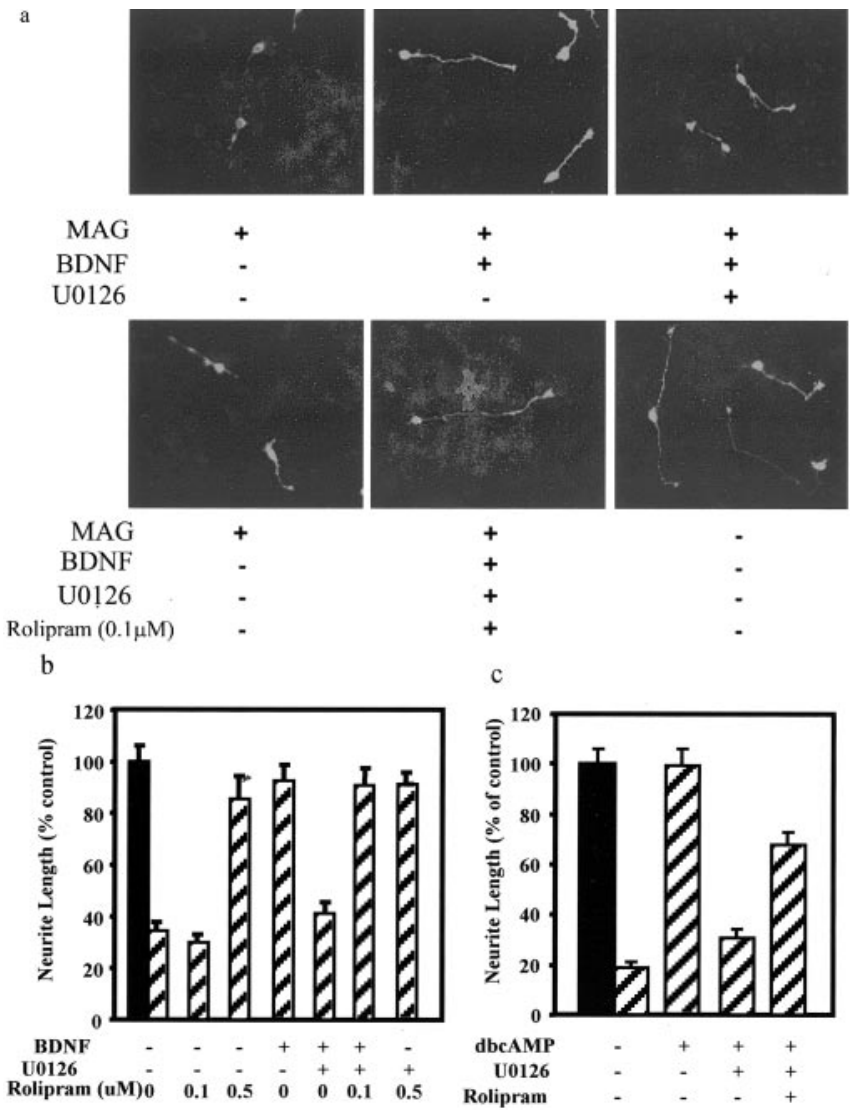

Figure 5. Low level, direct inhibition of PDE4 maintains the ability of BDNF to overcome inhibition by MAG in the presence of an Erk inhibitor. Cerebellar neurons were primed with and without $200 \mathrm{ng} / \mathrm{ml}$ BDNF before being transferred to control CHO cells (black bars) or MAGexpressing $\mathrm{CHO}$ cells (stripped bars). Where indicated, the PDE4 inhibitor rolipram (0.1 or 0.5 $\mu \mathrm{m})$ and/or the MEK inhibitor U0126 (5 $\mu \mathrm{m})$ were included during priming. After overnight culture, the cells were fixed and immunostained for GAP43 $(a)$. Results are the mean \pm SEM length of the longest neurite per neuron for 180-200 individual neurons, standardized to percentage of control $(b)$. Each experiment was performed in duplicate and repeated at least three times.

Direct inhibition of PDE maintains the ability of BDNF and 1 mM db-cAMP to overcome inhibition of MAG in the presence of MEK inhibitors

In non-neuronal cells, activated Erk has been shown to inhibit a subfamily of PDEs, the PDE4s, resulting in an increase in cAMP (Hoffmann et al., 1999; Baillie et al., 2000; MacKenzie et al., 2000). We reasoned that if MEK-Erk activation was having its effect through inhibition of PDE, then simultaneous, direct inhibition of PDE should block the effect of MEK-Erk inhibitors on inhibition by MAG. The drug rolipram is a specific inhibitor of PDE4, the most abundant PDE in neurons (Houslay and Kolch, 2000). When neurons are primed with rolipram at a concentration of $0.5 \mu \mathrm{M}$, inhibition by MAG is blocked; results are comparable with priming with BDNF (Fig. $5 a, b)$ ). In contrast, at a concentration of $0.1 \mu \mathrm{M}$, rolipram has no effect on inhibition (Fig. $5 a, b)$. However, when rolipram at $0.1 \mu \mathrm{M}$ is included in the cultures with U0126, during priming with BDNF, or when added directly to the cultures with $1 \mathrm{mM}$ db-cAMP, the ability to overcome inhibition is maintained (Fig. $4 a, b$ ). Similar results were obtained when another reagent that elevates cAMP, forskolin, was used. Forskolin overcomes inhibition by MAG in a dosedependent manner, and inhibition is completely blocked at $1 \mu \mathrm{M}$. However, as with db-cAMP and BDNF, U0126 blocks the effect a

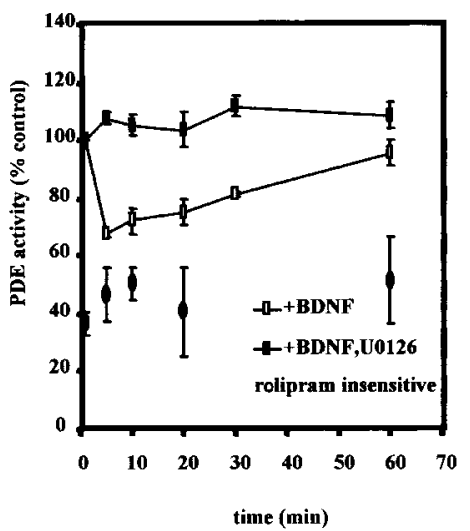

b

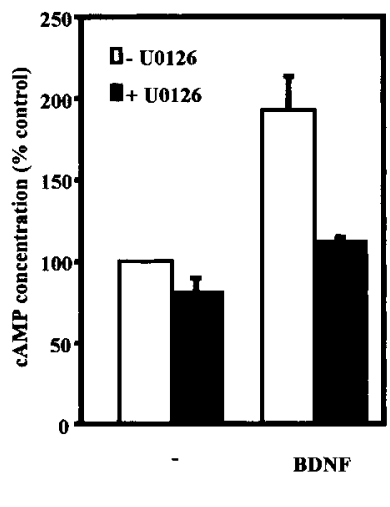

Figure 6. BDNF induces a transient inhibition of PDE4 and increases CAMP in an Erkdependent manner. $a$, Cerebellar neurons $\left(5 \times 10^{6}\right)$ were incubated with $200 \mathrm{ng} / \mathrm{ml}$ BDNF alone (open squares) or in the presence of $5 \mu \mathrm{m}$ U0126 (filled squares) for various times before lysing, or $100 \mu \mathrm{m}$ rolipram was added to the lysates (filled circles) and PDE activity was measured. Results are expressed as a percentage of PDE activity in untreated neurons \pm SE. For each experiment, samples were in triplicate and the experiment was repeated at least three times. $b$, cAMP levels were measured using a competitive immunoassay after plating $2 \times 10^{6}$ dissociated cerebellar neurons per well and treating with and without $200 \mathrm{ng} / \mathrm{mlBDNF}$, in the presence (dark bars) or absence (white bars) of U0126 for $30 \mathrm{~min}$. Results are the mean \pm SE of at least six experiments, each performed in quadruplicate.

of forskolin, and low-dose rolipram maintains it (results not shown). These results suggest that inhibition of MEK-Erk in the presence of BDNF, db-cAMP, or forskolin pushes the level of cAMP to just below the threshold required to overcome inhibition by MAG. Low concentrations of rolipram, however, counteract the effect of Erk inhibition and maintain the levels of cAMP above threshold.

\section{BDNF inhibits PDE activity and elevates cAMP in an}

\section{Erk-dependent manner}

To demonstrate that BDNF affects PDE activity directly, cerebellar neurons were treated with BDNF, with and without the MEK inhibitor U0126. After various lengths of time, the cells were lysed and the PDE activity was measured. As can be seen in Figure $6 a$, within 5 min of treatment with BDNF, the PDE activity in cerebellar neurons has decreased by $\sim 35 \%$, a decrease that is then sustained for almost $1 \mathrm{hr}$. In sharp contrast, when the neurons are exposed to BDNF but in the presence of U0126, there is no decrease in the PDE activity; activity is the same as in control, untreated neurons. However, this assay measures total PDE activity. As reported by others, we show that $\sim 70-80 \%$ of the activity in neurons is rolipram-sensitive, PDE4 activity. The $20-30 \%$ nonPDE4 activity (rolipram insensitive) does not decrease in the presence of BDNF. Therefore, the decrease in PDE activity in response to BDNF is entirely attributable to an effect on PDE4. It follows that treatment of neurons with BDNF results in an inhibition of PDE4, and that an MEK inhibitor blocks this effect.

We have shown previously that BDNF increases the endogenous levels of cAMP in cerebellar neurons (Cai et al., 1999). Now we wanted to determine whether this increase in cAMP is MEKErk dependent. Cerebellar neurons were treated with BDNF with and without U0126. As can be seen in Figure $6 b$ and as we reported previously, after BDNF treatment the level of cAMP in 
cerebellar neurons almost doubles. However, when the MEK inhibitor U0126 is included along with BDNF, this increase in cAMP does not occur. Together, these results show (1) that BDNF can inhibit PDE4 activity and (2) that both the BDNFinduced inhibition of PDE4 and the subsequent increase in cAMP are MEK-Erk dependent.

\section{Discussion}

Although we have clearly shown previously that a variety of neurotrophins were able to overcome inhibition by MAG and myelin in a CAMP-PKA-dependent manner, activation of receptor tyrosine kinases is not classically regarded as involving the cAMP pathway in their signaling. Here we show that it is cross talk between two pathways that is responsible for the neurotrophininduced, Trk-dependent increase in cAMP that overcomes inhibition. As has been shown previously, Erk is activated in response to neurotrophin, but now rather than signaling directly to transcription factors such as cAMP response element-binding protein (CREB), we show that activated Erk also inhibits PDE, and thus CAMP levels increase and PKA is activated. To our knowledge, this is the first time that activation of Erk has been shown to activate cAMP-dependent pathways in neurons.

Our results also reveal that there is a threshold of CAMP-PKA activation required to overcome inhibition. For the neurotrophin effect, reaching the threshold is completely dependent on Erk activation. In contrast, inhibition of Erk only affects the ability of db-cAMP to overcome inhibition by MAG at lower concentrations; at higher db-cAMP concentrations, inhibition of Erk has no effect. We propose that at lower concentrations of db-cAMP, both the exogenous db-cAMP and the endogenous cAMP are together required to reach the threshold. That is to say, at $1 \mathrm{mM}$ $\mathrm{db}$-cAMP, after inhibition of Erk, basal inhibition of PDE is relieved and endogenous cAMP levels decrease, and $1 \mathrm{~mm} \mathrm{db}$ cAMP alone does not reach the threshold of activation. At $2 \mathrm{mM}$ $\mathrm{db}$-cAMP, the threshold is reached regardless of whether Erk is inhibited. From these observations, it follows that there must be a threshold of activation of signals downstream from cAMP elevation. Consistent with this, we have shown that activation of PKA increases as the db-cAMP concentration is increased, and that inhibition of Erk lowers the PKA activity at $1 \mathrm{mM} \mathrm{db}$-cAMP but has no effect at $2 \mathrm{~mm} \mathrm{db}$-cAMP; at $2 \mathrm{~mm} \mathrm{db}$-cAMP, PKA is activated above threshold, and endogenous cAMP is not required. The next question is what is downstream from PKA activation? We have already established that the effects of both neurotrophin and db-cAMP are transcription dependent. It is possible, then, that a threshold of activation of transcription factors such as CREB is, in turn, required to overcome inhibition.

These results are important for a number of reasons. First, stimulation of the CAMP pathway is a novel signaling mechanism for neurotrophins. Other effects of neurotrophins, such as survival, differentiation, and neurite outgrowth, are regarded as cAMP-PKA independent (Rydel and Greene, 1988; Damon et al., 1990; Boniece and Wagner, 1993). It should be noted that in the experiments described here, neurotrophins are not simply promoting neurite extension but are overcoming inhibitors in myelin. It is possible that signaling through the cAMP pathway may contribute to effects of neurotrophins that cannot be explained simply by a transient activation of Erk (see below). Previous studies using PC12 cells showed that NGF elevated cAMP within seconds, but not through TrkA (Knipper et al., 1993a,b). This effect is very different from what we report here, because the effect we report is over a much longer time frame (minutes to hours) and is through Trk. Furthermore, no functional relevance has been attributed to this rapid increase in cAMP in PC12 cells in response to NGF. Second, molecular dissection of the signaling pathway that results in neurotrophins overcoming inhibition will allow us to manipulate the pathway more specifically to overcome inhibition and encourage regeneration. This in turn may reveal an approach whereby the pathway can be stimulated more directly and so obviate the need to prime. Third, the results with both BDNF and db-cAMP indicate that there is a set activation threshold for cAMP that is required to overcome inhibition. The threshold of activation of CAMP-PKA, and perhaps of downstream effectors, may be different for different responses. This may explain how the same signaling pathway can have a variety of effects. Fourth, it has been established that cyclic nucleotides can modulate the response of growth cones to guidance cues. Because we show here that neurotrophins can modulate cAMP, it follows that they may also modulate the response to guidance cues.

As reported here and by others (Stork and Schmitt, 2002), high levels of cAMP can activate Erk. This is suggested to occur when elevated cAMP activates the cAMP-dependent, PKAindependent G-protein exchange factors Epac-1 and Epac-2, which in turn activate the Rap-1-B-Raf pathway, resulting in sustained activation of Erk (Grewal et al., 1999; de Rooij et al., 2000). Furthermore, the kinetics of Erk activation in PC12 cells in response to NGF is consistent with consecutive activation of two pathways. Within the first $2-5$ min of NGF treatment, Erk is activated through the Ras-c-Raf pathway, after which it becomes independent of this pathway but dependent for further activation on the Rap-1-B-Raf pathway (York et al., 1998; Wu et al., 2001). The mechanism of this switch in pathways that activate Erk in response to a single stimulus is not yet known. The mechanism we describe here, whereby transient inhibition of PDE4 by Erk, subsequent to Trk activation, leads to a transient increase in cAMP, could explain the switch from Erk activation in a Ras-cRaf-dependent manner to activation in a Rap-1-B-Rafdependent, Ras-c-Raf-independent manner.

It is of note that for certain CNS neurons, elevation of cAMP has been shown to potentiate neurotrophin-induced survival effects (Meyer-Franke et al., 1995; Hanson et al., 1998; MeyerFranke et al., 1998). Bidirectional cross talk between Erk and cAMP signaling could explain this effect. Furthermore, these enhanced survival effects resulting from a combination of neurotrophin and cAMP suggest that here too, as with overcoming inhibition by MAG, a threshold of cAMP must be reached and/or prolonged activation of Erk is required.

Recently the p75NTR was shown to be part of a receptor complex that transduces inhibition by MAG, OMgp, and Nogo66 (Wang et al., 2002a; Wong et al., 2002). On the basis of a number of results, we propose that p75NTR is not involved in the neurotrophin priming effect. First, for cerebellar neurons that express p75NTR but not TrkA (Martin-Zanca et al., 1990; Holtzman et al., 1992), priming with NGF has no effect on inhibition by MAG. Second, a kinase inhibitor known to block Trk signaling blocks the priming effect completely Third, an antibody that prevents neurotrophin binding to p75NTR (Huber and Chao, 1995) has no effect on the ability of DRG neurons primed with NGF or BDNF to overcome inhibition. These findings present an interesting scenario in which two receptors that bind the same ligands 
are involved in opposing effects, bringing about and overcoming inhibition of neurite outgrowth by MAG and myelin.

The question remains: why must neurons be primed with neurotrophins to overcome inhibition? As exemplified in the studies described here, no signaling pathway operates in isolation, but rather through cross talks with other pathways. Previously, we provided evidence that MAG and myelin activate a heterotrimeric $\mathrm{G}$-protein, $\mathrm{G}_{\mathrm{i}} / \mathrm{G}_{\mathrm{o}}$, which inhibits adenylate cyclase, the enzyme that synthesizes cAMP (Cai et al., 1999). In addition, $G_{i} / G_{o}$ has been shown to regulate the Rap-1 pathway (Johnson et al., 1989; Mochizuki et al., 1999). How $\mathrm{G}_{\mathrm{i}} / \mathrm{G}_{\mathrm{o}}$ activation by MAG-myelin intercepts the neurotrophin signaling pathway remains to be elucidated.

In summary, we propose a model whereby NGF-like neurotrophins, via Trk receptors, activate Erk and transiently inhibit $\mathrm{PDE}$ and so can elevate cAMP to overcome inhibition of regeneration by MAG. We also demonstrate that there is a threshold of activation of cAMP-PKA for both db-cAMP and neurotrophins to overcome inhibition. Further dissection of this pathway may lead to a more focused approach to encourage regeneration in vivo.

\section{References}

Baillie GS, MacKenzie SJ, McPhee I, Houslay MD (2000) Sub-family selective actions in the ability of Erk2 MAP kinase to phosphorylate and regulate the activity of PDE4 cyclic AMP-specific phosphodiesterases. Br J Pharmacol 131:811-819.

Boniece IR, Wagner JA (1993) Growth factors protect PC12 cells against ischemia by a mechanism that is independent of PKA, PKC, and protein synthesis. J Neurosci 13:4220-4228.

Bouschet T, Perez V, Fernandez C, Bockaert J, Eychene A, Journot L (2003) Stimulation of the ERK pathway by GTP-loaded Rap1 requires the concomitant activation of Ras, protein kinase $\mathrm{C}$, and protein kinase A in neuronal cells. J Biol Chem 278:4778-4785.

Cai D, Shen Y, De Bellard M, Tang S, Filbin MT (1999) Prior exposure to neurotrophins blocks inhibition of axonal regeneration by MAG and myelin via a cAMP-dependent mechanism. Neuron 22:89-101.

Chen MS, Huber AB, van der Haar ME, Frank M, Schnell L, Spillmann AA, Christ F, Schwab ME (2000) Nogo-A is a myelin-associated neurite outgrowth inhibitor and an antigen for monoclonal antibody IN-1. Nature 403:434-439.

Damon DH, D'Amore PA, Wagner JA (1990) Nerve growth factor and fibroblast growth factor regulate neurite outgrowth and gene expression in PC12 cells via both protein kinase C- and cAMP-independent mechanisms. J Cell Biol 110:1333-1339.

de Rooij J, Rehmann H, van Triest M, Cool RH, Wittinghofer A, Bos JL (2000) Mechanism of regulation of the Epac family of cAMP-dependent RapGEFs. J Biol Chem 275:20829-20836.

Domeniconi M, Cao Z, Spencer T, Sivasankaran R, Wang RC, Nikulina E, Kimura N, Cai H, Deng K, Gao Y, He Z, Filbin MT (2002) Myelinassociated glycoprotein interacts with the Nogo66 receptor to inhibit neurite outgrowth. Neuron 35:283-290.

Filbin MT (2003) Myelin-associated inhibitors of axonal regeneration in the adult mammalian CNS. Nat Rev Neurosci 4:703-713.

Fitch MT, Silver J (1999) Beyond the glial scar. In: CNS regeneration (Tuszynski MH, Kordower JH, eds), pp 55-88. San Diego: Academic.

Fournier AE, GrandPre T, Strittmatter SM (2001) Identification of a receptor mediating Nogo-66 inhibition of axonal regeneration. Nature 409:341-346.

GrandPre T, Nakamura F, Vartanian T, Strittmatter SM (2000) Identification of the Nogo inhibitor of axon regeneration as a reticulon protein. Nature 403:439-444.

Grewal SS, York RD, Stork PJ (1999) Extracellular-signal-regulated kinase signalling in neurons. Curr Opin Neurobiol 9:544-553.

Hanson Jr MG, Shen S, Wiemelt AP, McMorris FA, Barres BA (1998) cAMP elevation is sufficient to promote the survival of spinal motor neurons in vitro. J Neurosci 18:7361-7371.

He TC, Zhou S, da Costa LT, Yu J, Kinzler KW, Vogelstein B (1998) A simplified system for generating recombinant adenoviruses. Proc Natl Acad Sci USA 95:2509-2514.

Hoffmann R, Baillie GS, MacKenzie SJ, Yarwood SJ, Houslay MD (1999) The MAP kinase ERK2 inhibits the cyclic AMP-specific phosphodiesterase HSPDE4D3 by phosphorylating it at Ser579. EMBO J 18:893-903.

Holtzman DM, Li Y, Parada LF, Kinsman S, Chen CK, Valletta JS, Zhou J, Long JB, Mobley WC (1992) p140trk mRNA marks NGF-responsive forebrain neurons: evidence that trk gene expression is induced by NGF. Neuron 9:465-478.

Houslay MD, Kolch W (2000) Cell-type specific integration of cross-talk between extracellular signal-regulated kinase and cAMP signaling. Mol Pharmacol 58:659-668.

Huber LJ, Chao MV (1995) A potential interaction of p75 and trkA NGF receptors revealed by affinity crosslinking and immunoprecipitation. J Neurosci Res 40:557-563.

Jin SL, Richard FJ, Kuo WP, D’Ercole AJ, Conti M (1999) Impaired growth and fertility of cAMP-specific phosphodiesterase PDE4D-deficient mice. Proc Natl Acad Sci USA 96:11998-12003.

Johnson PW, Abramow-Newerly W, Seilheimer B, Sadoul R, Tropak MB, Arquint M, Dunn RJ, Schachner M, Roder JC (1989) Recombinant myelin-associated glycoprotein confers neural adhesion and neurite outgrowth function. Neuron 3:377-385.

Kaplan DR, Miller FD (2000) Neurotrophin signal transduction in the nervous system. Curr Opin Neurobiol 10:381-391.

Knipper M, Beck A, Rylett J, Breer H (1993a) Neurotrophin induced cAMP and IP3 responses in PC12 cells. Different pathways. FEBS Lett 324:147-152.

Knipper M, Beck A, Rylett J, Breer H (1993b) Neurotrophin induced second messenger responses in rat brain synaptosomes. NeuroReport 4:483-486.

Lehmann M, Fournier A, Selles-Navarro I, Dergham P, Sebok A, Leclerc N, Tigyi G, McKerracher L (1999) Inactivation of Rho signaling pathway promotes CNS axon regeneration. J Neurosci 19:7537-7547.

Liu BP, Fournier A, GrandPre T, Strittmatter SM (2002) Myelin-associated glycoprotein as a functional ligand for the Nogo-66 receptor. Science 297:1190-1193.

MacKenzie SJ, Baillie GS, McPhee I, Bolger GB, Houslay MD (2000) ERK2 mitogen-activated protein kinase binding, phosphorylation, and regulation of the PDE4D cAMP-specific phosphodiesterases. The involvement of $\mathrm{COOH}$-terminal docking sites and NH2-terminal UCR regions. J Biol Chem 275:16609-16617.

Martin-Zanca D, Barbacid M, Parada LF (1990) Expression of the trk protooncogene is restricted to the sensory cranial and spinal ganglia of neural crest origin in mouse development. Genes Dev 4:683-694.

McKerracher L, David S, Jackson DL, Kottis V, Dunn RJ, Braun PE (1994) Identification of myelin-associated glycoprotein as a major myelinderived inhibitor of neurite growth. Neuron 13:805-811.

Meyer-Franke A, Kaplan MR, Pfrieger FW, Barres BA (1995) Characterization of the signaling interactions that promote the survival and growth of developing retinal ganglion cells in culture. Neuron 15:805-819.

Meyer-Franke A, Wilkinson GA, Kruttgen A, Hu M, Munro E, Hanson Jr MG, Reichardt LF, Barres BA (1998) Depolarization and cAMP elevation rapidly recruit TrkB to the plasma membrane of CNS neurons. Neuron 21:681-693.

Mochizuki N, Ohba Y, Kiyokawa E, Kurata T, Murakami T, Ozaki T, Kitabatake A, Nagashima K, Matsuda M (1999) Activation of the ERK/ MAPK pathway by an isoform of rap1GAP associated with $\mathrm{G} \alpha(\mathrm{i})$. Nature 400:891-894.

Mukhopadhyay G, Doherty P, Walsh FS, Crocker PR, Filbin MT (1994) A novel role for myelin-associated glycoprotein as an inhibitor of axonal regeneration. Neuron 13:757-767.

Neumann S, Bradke F, Tessier-Lavigne M, Basbaum AI (2002) Regeneration of sensory axons within the injured spinal cord induced by intraganglionic cAMP elevation. Neuron 34:885-893.

Qiu J, Cai D, Filbin MT (2000) Glial inhibition of nerve regeneration in the mature mammalian CNS. Glia 29:166-174.

Qiu J, Cai D, Dai H, McAtee M, Hoffman PN, Bregman BS, Filbin MT (2002) Spinal axon regeneration induced by elevation of cyclic AMP. Neuron 34:895-903.

Robinson MJ, Harkins PC, Zhang J, Baer R, Haycock JW, Cobb MH, Goldsmith EJ (1996) Mutation of position 52 in ERK2 creates a nonproduc- 
tive binding mode for adenosine $5^{\prime}$-triphosphate. Biochemistry 35:5641-5646.

Rydel RE, Greene LA (1988) cAMP analogs promote survival and neurite outgrowth in cultures of rat sympathetic and sensory neurons independently of nerve growth factor. Proc Natl Acad Sci USA 85:1257-1261.

Song H, Ming G, He Z, Lehmann M, McKerracher L, Tessier-Lavigne M, Poo M (1998) Conversion of neuronal growth cone responses from repulsion to attraction by cyclic nucleotides. Science 281:1515-1518.

Stork PJ, Schmitt JM (2002) Crosstalk between cAMP and MAP kinase signaling in the regulation of cell proliferation. Trends Cell Biol 12:258-266.

Vossler MR, Yao H, York RD, Pan MG, Rim CS, Stork PJ (1997) cAMP activates MAP kinase and Elk-1 through a B-Raf- and Rap1-dependent pathway. Cell 89:73-82.
Wang KC, Kim JA, Sivasankaran R, Segal R, He Z (2002a) P75 interacts with the Nogo receptor as a co-receptor for Nogo, MAG, and OMgp. Nature 420:74-78.

Wang KC, Koprivica V, Kim JA, Sivasankaran R, Guo Y, Neve RL, He Z (2002b) Oligodendrocyte-myelin glycoprotein is a Nogo receptor ligand that inhibits neurite outgrowth. Nature 417:941-944.

Wong ST, Henley JR, Kanning KC, Huang KH, Bothwell M, Poo MM (2002) A p75(NTR) and Nogo receptor complex mediates repulsive signaling by myelin-associated glycoprotein. Nat Neurosci 5:1302-1308.

Wu C, Lai CF, Mobley WC (2001) Nerve growth factor activates persistent Rap1 signaling in endosomes. J Neurosci 21:5406-5416.

York RD, Yao H, Dillon T, Ellig CL, Eckert SP, McCleskey EW, Stork PJ (1998) Rap1 mediates sustained MAP kinase activation induced by nerve growth factor. Nature 392:622-626. 